\title{
The CXCL13 chemokine serves as a potential biomarker to diagnose systemic lupus erythaematosus with disease activity
}

\author{
yanli zeng ${ }^{1}$, Yan Zhang ${ }^{1}$, Yiqiang Lin $^{1}$, Xuelian Wang ${ }^{2}$, Qinggui Chen ${ }^{3}$, Qinghe Huang ${ }^{4}$, \\ Jiajia Wang ${ }^{1}$, Longcan Jiang $^{1}$, and Yun Xiao ${ }^{1}$ \\ ${ }^{1}$ Center of Clinical Laboratory, Zhongshan Hospital, School of Medicine, Xiamen University \\ ${ }^{2}$ Department of Obstetrics and Gynecology, Zhongshan Hospital of Xiamen University \\ ${ }^{3}$ Department of general surgery, Zhongshan Hospital of Xiamen University \\ ${ }^{4}$ Department of Intensive Care Unit, Zhongshan Hospital of Xiamen University
}

October 30, 2020

\begin{abstract}
Objectives: Our study purpose was to assess the regulatory response of the chemokine CXCL13 in the serum of patients with systemic lupus erythaematosus (SLE) and to evaluate its influence on the inflammatory process in SLE. Methods: Serum samples from 97 SLE patients, 49 non-SLE patients (23 patients with other autoimmune diseases and 26 patients with rheumatoid arthritis ) and 50 healthy controls were analysed for the concentration of CXCL13 using ELISA. Results: The results indicated that the serum levels of CXCL13 were significantly higher in SLE patients than in non-SLE patients and healthy controls $(\mathrm{p}<0.001)$. Moreover, the level of CXCL13 decreased as the level of anti-dsDNA IgG decreased after treatment between the anti-dsDNA-positive SLE patients and the anti-dsDNA-negative SLE patients. In addition, serum CXCL13 levels were correlated with SLEDAI in different activities of SLE, renal involvement and active LN. Furthermore, the level of CXCL13 was positively related to the SLEDAI,level of anti-dsDNA IgG, level of ESR and RAI of high-avidity IgG ANAs (HA IgG ANAs). Additionally, ROC curve analysis revealed that the serum CXCL13 levels were robust in discriminating patients with active SLE from patients with inactive SLE and SLE patients with high-avidity IgG ANAs from SLE patients with low-avidity IgG ANAs. Conclusions: First, we demonstrated that CXCL13 was elevated in SLE patients regardless of the presence or absence of anti-dsDNA IgG ANAs. Furthermore, HA IgG ANAs might affect the circulation of CXCL13. Therefore, the chemokine CXCL13 might be a risk factor influencing the inflammatory process in SLE.
\end{abstract}

The CXCL13 chemokine serves as a potential biomarker to diagnose systemic lupus erythaematosus with disease activity

Yanli Zeng ${ }^{1,}{ }^{*}$, Yan Zhang ${ }^{1,}{ }^{*}$, Yiqiang $\operatorname{Lin}^{1}{ }^{*}$, Xuelian $\mathrm{Wang}^{2}$, Qinggui Chen ${ }^{3}$, Qinghe Huang ${ }^{4}$, JiaJia Wang ${ }^{1}$, Longcan Jiang ${ }^{1}$, Yun Xiao ${ }^{1}$

Yanli Zeng, Yan Zhang,Yiqiang Lin,JiaJia Wang,Longcan Jiang, and Yun Xiao

${ }^{1}$ Center of Clinical Laboratory, Zhongshan Hospital, School of Medicine, Xiamen University, Xiamen, China, 361004.

2 Department of Obstetrics and Gynecology, Zhongshan Hospital of Xiamen University, Xiamen, China, 361004 .

${ }^{3}$ Department of general surgery, Zhongshan Hospital of Xiamen University, Xiamen, China, 361004.

${ }^{4}$ Department of Intensive Care Unit, Zhongshan Hospital of Xiamen University, Xiamen, China, 361004.

*These authors contributed equally to this work. 
corresponding author, email address:xiaoyun@xmu.edu.cn Yun Xiao corresponding author. Tel.: +86-5922993046 Fax: +86-592-2993043

\begin{abstract}
Objectives: Our study purpose was to assess the regulatory response of the chemokine CXCL13 in the serum of patients with systemic lupus erythaematosus (SLE) and to evaluate its influence on the inflammatory process in SLE.
\end{abstract}

Methods: Serum samples from 97 SLE patients, 49 non-SLE patients (23 patients with other autoimmune diseases and 26 patients with rheumatoid arthritis ) and 50 healthy controls were analysed for the concentration of CXCL13 using ELISA.

Results: The results indicated that the serum levels of CXCL13 were significantly higher in SLE patients than in non-SLE patients and healthy controls $(p<0.001)$. Moreover, the level of CXCL13 decreased as the level of anti-dsDNA IgG decreased after treatment between the anti-dsDNA-positive SLE patients and the anti-dsDNA-negative SLE patients. In addition, serum CXCL13 levels were correlated with SLEDAI in different activities of SLE, renal involvement and active LN. Furthermore, the level of CXCL13 was positively related to the SLEDAI,level of anti-dsDNA IgG, level of ESR and RAI of high-avidity IgG ANAs (HA IgG ANAs). Additionally, ROC curve analysis revealed that the serum CXCL13 levels were robust in discriminating patients with active SLE from patients with inactive SLE and SLE patients with high-avidity IgG ANAs from SLE patients with low-avidity IgG ANAs.

Conclusions: First, we demonstrated that CXCL13 was elevated in SLE patients regardless of the presence or absence of anti-dsDNA IgG ANAs. Furthermore, HA IgG ANAs might affect the circulation of CXCL13. Therefore, the chemokine CXCL13 might be a risk factor influencing the inflammatory process in SLE.

Key words: B-cell-attracting chemokine, CXC ligand 13 (CXCL13), high-avidity IgG ANAs, systemic lupus erythaematosus, lupus nephritis(LN)

\title{
Key points
}

The following points are the key points of this manuscript:

1. The serum levels of CXCL13 were significantly higher in SLE patients than controls.

2. We suggested that high-avidity IgG ANAs might affect the circulation of CXCL13.

3. The chemokine CXCL13 might be a risk factor influencing the inflammatory process in SLE.

\section{Introduction}

Systemic lupus erythaematosus (SLE) is a multisystem autoimmune disease characterized by the presence of a large number of autoantibodies and a multitude of immune complex deposits in the target tissues. Antinuclear antibodies (ANAs) are a type of specific autoantibody that has been evaluated for the diagnosis of systemic autoimmune rheumatic diseases (SARDs) and SLE in clinical work since the $1940 \mathrm{~s}^{1,2}$. Antidouble-stranded DNA (dsDNA) autoantibodies are important laboratory biomarkers associated with disease activity in $\mathrm{SLE}^{3}$. The combined detection of ANAs and anti-dsDNA can improve the diagnostic specificity, sensitivity and disease activity of SLE, which compensates for the titre of ANAs that remain constant for a certain period of time. Although anti-dsDNA antibodies show high SLE specificity, their prevalence in different studies has been found to be only in 50\% of SLE patients and does not always correlate with disease activity $^{4,5}$. Likewise, we found that approximately $27.8 \%$ (27/97) of SLE patients were anti-dsDNA-negative, although patients' clinical symptoms were active in our study.

Previous studies have shown that the B-cell-attracting chemokine CXC ligand 13 (CXCL13) is closely related to autoimmune diseases, especially SLE, rheumatoid arthritis (RA), Sjögren's syndrome and other autoimmune diseases ${ }^{6-10}$. CXCL13 is significantly increased in SLE patients, especially in lupus nephritis (LN) patients and is positively correlated with the SLE disease activity index (SLEDAI) and anti-dsDNA titre. The diagnostic sensitivity and specificity of LN are up to $95 \%$ and $88 \%$, respectively ${ }^{9}$. However, the 
involvement of CXCL13 in SLE patients who are anti-dsDNA-negative still needs to be investigated further. Here, we assessed the regulatory response of CXCL13 in the serum of SLE anti-dsDNA-positive patients, SLE anti-dsDNA-negative patients, non-SLE patients and healthy controls. The changes in CXCL13 in the SLE patients after treatment were studied to evaluate the effects of CXCL13. In addition, the correlations between CXCL13 and clinical disease activities and laboratory results were studied to examine the possible mechanisms of CXCL13.

\section{Materials and methods}

\subsection{Patients and controls}

The study was approved by the Research Ethics Committee of Zhongshan Hospital, the Medical College of Xiamen University, and informed consent was obtained from all the participants. Among all participants, pregnant women and patients with various cancers and known defects of the immune system (such as HIV infection, haematologic diseases or transplantation history) were excluded.

SLE group : A total of 97 patients with SLE (8 males and 89 females, median age of 34 years) diagnosed according to the 2012 revisited diagnostic criteria of the European League against Rheumatism (EULAR) and the American College of Rheumatology criteria were accepted from Zhongshan Hospital, the Medical College of Xiamen University, from April 2019 to June 2020. In this study, we subdivided the patients in the SLE group into two groups based on the presence or absence of anti-dsDNA antibodies (anti-dsDNA+ and anti-dsDNA- groups) before treatment. The disease activity of SLE patients was evaluated according to the SLE disease activity index 2000 (SLEDAI-2K), and SLEDAI-2K >4 was defined as active SLE ${ }^{11,12}$. Patients who were diagnosed with lupus nephritis (LN) were evaluated using urine analysis, renal function tests, and renal biopsies, as previously described ${ }^{13}$.

Non-SLE group : This group was composed of two subgroups, the rheumatoid arthritis (RA) group and the other autoimmune diseases (OAD) group. Twenty-six patients (7 males and 19 females, median age of 31.5 years) were diagnosed with RA according to the 2010 American College of Rheumatology (ACR)/European League Against Rheumatism (EULAR) rheumatoid arthritis (RA) classification criteria (2010 RA criteria) ${ }^{14}$. Twenty-three patients ( 5 males and 18 females, median age of 36 years) were recruited with other autoimmune diseases. Of these patients, 13 were diagnosed with Sjögren's syndrome (SS), 6 were diagnosed with mixed connective tissue disease (MCTD), 3 were diagnosed with dermatomyositis, and 1 was diagnosed with antiphospholipid syndrome (APS).

Healthy control group: This group was composed of 50 age- and sex-matched individuals (10 males and 40 females, median age of 31 years) without any risk factors or chronic diseases.

The most common medicine used by SLE patients was prednisone (90/97, 92.8\%), and some SLE patients were treated with prednisone combined with chloroquine or chloroquine plus azathioprine (64/97, 66.0\%). Methotrexate plus prednisone and chloroquine was used in fewer patients $(28 / 97,28.9 \%)$ than other medicine. Cyclophosphamide was rarely used $(10 / 97,10.30 \%)$.

2.2 Detection of total IgG ANAs, anti-dsDNA antibodies, and the avidities of IgG ANAs and anti-CXCL13 antibodies

The serum level of total IgG ANA levels and concentration of anti-dsDNA were measured by enzymelinked immunosorbent assay (ELISA) according to the manufacturer's protocol and our previous studies ${ }^{15}$. The avidity of IgG ANAs by ELISA and indirect immunofluorescence (IIF) assay were processed as in the standard test, according to the manufacturer's instructions and our previous studies. The relative avidity index (RAI) was used to evaluate the avidity of IgG ANAs.

The concentration of CXCL13 in serum was measured using human CXCL13/BLC/BCA-1 ELISA kits (CUSABIO, Wuhan, China) according to the manufacturer's instructions and as described in our previous studies $^{16}$. 
The serum levels of C3 and C4 were determined by nephelometry with the Image Immunochemistry System (Roche Cobas 8000, Germany).

\subsection{Statistical analysis}

The differences between patient groups and/or healthy controls were evaluated using the nonparametric Mann-Whitney U test. The correlations between CXCL13 concentrations and parameters of disease activity were calculated with Spearman's rank correlation. Receiver operator characteristic (ROC) curves showed optimal cut-off values for CXCL13 to differentiate between patients with active SLE and patients with inactive SLE and between SLE patients with high avidity of IgG ANAs and SLE patients with low-avidity IgG ANAs. The significance level was set to $p<0.05$. Data are displayed as the median \pm interquartile range (IQR) unless otherwise stated. All statistical analyses were performed using SPSS software (version 20.0; IBM Inc., New York, USA).

\section{Results}

\subsection{The characteristics and laboratory results in different tests of study populations}

As shown in Table 1, the prevalence of IgG ANAs and HA IgG ANAs, the levels of serum CXCL13, serum anti-dsDNA IgG, the complement molecules C3 and C4, and ESR were different among the SLE antidsDNA-positive group, the SLE anti-dsDNA-negative group, the non-SLE group and the healthy controls group $(p<0.001)$. Furthermore, we divided the non-SLE groups into the RA group and the other autoimmune diseases (OAD) group. Significant increases in the levels of CXCL13 were observed in the SLE anti-dsDNA+ group [487.70 $(266.94-928.74) \mathrm{pg} / \mathrm{ml}]$ among the other four groups $(p<0.05)$. The serum CXCL13 level was significantly higher in the SLE anti-dsDNA-negative group [213.95 (43.49-475.37) pg/ml], RA group [98.49 (39.07-249.05) pg/ml] and other autoimmune diseases group [246.73 $(117.04-451.56) \mathrm{pg} / \mathrm{ml}]$ than in the healthy controls group [0.045 $(0.01-15.17)](p<0.001)$. However, there were no differences in the serum CXCL13 levels among the SLE anti-dsDNA-negative group, RA group and OAD group. Moreover, the serum levels of C3 and C4 were significantly lower in the SLE anti-dsDNA-positive group than in the other four groups $(p<0.05)$. However, significant increases in the erythrocyte sedimentation rate (ESR) were observed in the RA group compared to that in the other groups, except the SLE anti-dsDNA-positive group $(p<0.05)$.

3.2 Comparisons of serum concentrations of CXCL13 and anti-dsDNA IgG levels before and after treatment between the SLE anti-dsDNA+ group and SLE anti-dsDNA- group

In our study, the serum levels of CXCL13 were examined before and after treatment between 47 patients in the SLE anti-dsDNA-positive group and 16 patients in the SLE anti-dsDNA-negative group. We found the same tendency towards a significant decrease in the serum levels of CXCL13 after treatment in both groups $(p<0.05)$ (Figure 1a, b). Furthermore, we assessed the differences in the levels of CXCL13 and antidsDNA IgG before and after treatment in the SLE anti-dsDNA-positive group. The results showed the same decreasing tendency and a significant decrease in both the CXCL13 and anti-dsDNA levels after treatment $(p<0.001)$ (Figure 1c, d).

3.3 Associations of serum concentration of CXCL13 in different disease activities in SLE patients, LN/non-LN patients, active/inactive LN patients, and high-avidity/low-avidity IgG ANAs

In our cohort of 97 SLE patients, a total of 20 patients $(20.6 \%, 20 / 97)$ were diagnosed with no/mild disease activity SLE (0[?]SLEDAI[?]9), 44 patients $(45.4 \%, 44 / 97)$ were diagnosed with moderate disease activity SLE (10[?]SLEDAI[?]14), and 33 patients $(34.0 \%, 33 / 97)$ were diagnosed with severe disease activity SLE (SLEDAI[?]15). A total of 54 patients $(55.7 \%, 54 / 97)$ were diagnosed with LN, and $73(75.3 \%, 73 / 97)$ patents were diagnosed with high-avidity IgG ANAs of SLE. Significantly higher serum CXCL13 levels were observed in SLE patients with severe disease activity [1023.077 (648.565-1768.138) pg/ml] among the no/mild disease activity [212.523 (30.229-297.550) pg/ml] and moderate disease activity SLE [341.845 (222.341-514.179) $\mathrm{pg} / \mathrm{ml}$ groups $(p<0.001)$. (Figure $2 \mathrm{a})$. We also found that the serum CXCL13 levels were significantly higher in the LN group [609.746 (338.472-1101.573) pg/ml] and active LN group [683.145 (399.065-1205.628) 
$\mathrm{pg} / \mathrm{ml}]$ than in the non-LN group [279.310 (115.381-482.276) pg/ml] and inactive LN group [251.475 (125.930$315.340) \mathrm{pg} / \mathrm{ml}](p<0.001)$ (Figure $2 \mathrm{~b}, \mathrm{c})$. As in our previous study, we divided the SLE patients into a high-avidity IgG ANA group (RAI\%[?]40\%) and a low-avidity IgG ANA group (RAI\%<40\%) according our detection assay. We found that the serum CXCL13 level was significantly higher in the high-avidity IgG ANA group [590.915 (280.092-1045.527) pg/ml] than in the low-avidity IgG ANA group [266.978 (117.615-408.526) $\mathrm{pg} / \mathrm{ml}](p<0.001)$ (Figure 2d).

3.4 Correlation analysis of the serum levels of CXCL13 with the SLEDAI, the RAI (relative avidity index) of HA IgG ANAs, the serum levels of anti-dsDNA, the ESR, and the C3 and C4 complements in SLE patients

In SLE patients, there was a correlation among the level of CXCL13, disease activities and laboratory results. The results showed that the concentration of CXCL13 was positively correlated with the SLEDAI score and RAI of HA IgG ANAs $(\mathrm{r}=0.7547, p<0.0001 ; \mathrm{r}=0.5590, p<0.0001)$ (Figure 3a, b). The concentration of CXCL13 was positively related to the serum level of anti-dsDNA and ESR ( $\mathrm{r}=0.4860, p<0.0001 ; \mathrm{r}=0.3082, p$ $=0.0021$ ) (Figure 3c, d). Furthermore, the concentration of CXCL13 was negatively related and significantly decreased with serum C3 and C4 levels $(\mathrm{r}=-0.4605, p<0.0001 ; \mathrm{r}=-0.5165, p<0.0001)$ (Figure 3e, f).

3.5 CXCL13 as a potential differentiation marker of active SLE from inactive SLE and of high-avidity IgG ANAs from low-avidity IgG ANAs in SLE patients

To evaluate the diagnostic potential and discriminatory accuracy of the serum CXCL13 concentration for active SLE and high-avidity IgG ANAs in SLE, receiver operating characteristic (ROC) curve analysis and the corresponding AUC values were determined (Figure 4). ROC curve analysis revealed that the serum CXCL13 levels were robust in discriminating patients with active SLE from inactive SLE and from high-avidity IgG ANAs from low-avidity IgG ANAs in SLE patients, with AUC values of 0.832 (95\% CI 0.741-0.922, $p<$ $0.0001)$ and $0.762(95 \%$ CI $0.667-0.856, p=0.0001)$, respectively. The maximal Youden's index indicated that $150.27 \mathrm{pg} / \mathrm{mL}$ and $187.16 \mathrm{pg} / \mathrm{mL}$ were the best cut-off values to distinguish between active SLE and inactive SLE and between high-avidity IgG ANAs and low-avidity IgG ANAs in SLE patients, where the sensitivity and specificity were 89 and $90 \%, 86.3$ and $88 \%$, respectively.

\section{Discussion}

In this study, we have shown that serum CXCL13 levels were significantly higher in SLE patients, especially in the SLE anti-dsDNA-positive group. Moreover, we found the same tendency towards a significant decrease in the serum levels of CXCL13 after treatment between the SLE anti-dsDNA-positive group and the SLE anti-dsDNA-negative group. Furthermore, the SLE patients with severe disease activity had median levels of CXCL13 that were more than three times as high as those with no/mild disease activity or moderate disease activity. In addition, the levels of CXCL13 were significantly elevated in the renal involvement group and active LN group. CXCL13 levels were positively correlated with SLEDAI, the levels of anti-dsDNA, and ESR. However, CXCL13 levels were negatively correlated with complement levels of C3 and C4. This is consistent with previous research ${ }^{7,9,17}$. We first introduced the concept of an "HA IgG ANA" in our previous report ${ }^{15}$. Of interest, we demonstrated that CXCL13 levels were significantly higher in the highavidity IgG ANA group than in the low-avidity IgG ANA group. Furthermore, the concentration of CXCL13 was positively correlated and significantly correlated with the RAI of HA IgG ANAs.

In our study, we enrolled the SLE group, RA group, other autoimmune diseases group and healthy control group as our research subjects at the same time. We subdivided the patients in the SLE group into two groups based on the presence and absence of anti-dsDNA (anti-dsDNA+ and anti-dsDNA- groups). The results presented here demonstrated that the levels of CXCL13 were significantly increased in the SLE antidsDNA-positive group compared with those in the other four groups. However, there were no differences in serum CXCL13 levels among the SLE anti-dsDNA-negative group, RA group and other autoimmune diseases group. Difference research has identified that SLE, RA, and Sjogren's syndrome are associated with the levels of CXCL13, as in previous studies ${ }^{18}$. Through cross-examination, we found more clearly that when CXCL13 coexisted with high levels of anti-dsDNA, the concentration of CXCL13 reached its peak value. 
These results indicate that CXCL13 may contribute somewhat to the production of anti-dsDNA antibodies. It is speculated that the production of CXCL13 by immune-activated cells may lead to the aggregation of antibody-producing B cells into the target organ, where destructive autoantibodies such as anti-dsDNA are released in large quantities, leading to significant tissue damage. This is in line with the results of a previous study $^{19}$. It is well known that SLE, RA and other autoimmune diseases are characterized by increased production of autoantibodies that are produced long before the onset of clinical symptoms, leading to more chronic and severe consequences. Our results showed that there was a correlation between CXCL13 levels and SLE as well as RA and other autoimmune diseases. It was recommended that anti-dsDNA-negative SLE, RA and other autoimmune disease patients have significantly increased serum CXCL13 levels compared with healthy controls, although previous studies have never discussed this topic before ${ }^{9}$. It was suggested that CXCL13 plays a key role in the differentiation of B lymphocytes into plasma cells to generate antibodies ${ }^{6}$. However, the disease activity and correlation factors between CXCL13 and RA and other autoimmune diseases should be further studied.

Moreover, we first evaluated the regulatory response of serum concentrations of CXCL13 and anti-dsDNA IgG levels before and after treatment between the SLE anti-dsDNA-positive group and the SLE anti-dsDNAnegative group. Although Lee and co-workers divided SLE patients into anti-dsDNA+ and anti-dsDNAgroups, they observed significantly different levels of CXCL13 between these groups before treatment ${ }^{8}$. Of note, CXCL13 serum levels remained significantly elevated in anti-dsDNA-negative SLE patients, probably indicating aberrant B cell trafficking, even in remission. We further found the same tendency towards a significant decrease in the serum levels of CXCL13 after treatment, which was the same as the tendency of the anti-dsDNA levels. The present results indicate that CXCL13 is of great value for the prognosis of SLE, especially in the absence of anti-dsDNA antibodies.

As previous studies report, serum CXCL13 levels were associated with disease activity by the SLE Disease Activity Index (SLEDAI) and active lupus nephritis ${ }^{20}$. Consistent with the results of previous research, our study also showed that serum CXCL13 levels were correlated with SLEDAI in different disease activities of SLE, and the concentration of CXCL13 was higher in patients with renal involvement and active LN disease. Additionally, high baseline serum CXCL13 levels identify recent onset of inflammation with better response to early aggressive treatment in SLE patients. The mechanism of CXCL13 and SLE or CXCL13 and LN has attracted more attention from many scholars. It is well known that different chemokines are involved in the pathogenesis of LN by coordinating the pro-inflammatory microenvironments, recruiting immune cell subsets into the kidney, and inducing local activation of immune effector cells ${ }^{21}$. Different studies have consistently demonstrated that CXCL13 induces B cell infiltration into the kidneys via its receptor CXCR5 and enhances albuminuria and organ damage ${ }^{22}$. However, its exact place within the mechanisms that lead to SLE remains to be defined.

As previously reported, serum circulation of CXCL13 is positively related to disease activity (SLEDAI) and the concentrations of anti-dsDNA in SLE patients, but CXCL13 is negatively related to the complement factor C3. Likewise, the serum concentration of CXCL13 positively correlated with the SLEDAI, anti-dsDNA levels and ESR but negatively correlated with the complement factors of C3 and C4 in SLE patients in the present study. Circulating CXCL13 is a suitable supplementary biomarker of SLE activity. Furthermore, we found that the serum CXCL13 levels were robust in discriminating patients with active and inactive SLE, with an AUC of $0.832(95 \%$ CI $0.741-0.922, P<0.0001)$, and the sensitivity and specificity were 89 and $90 \%$, respectively, according to the ROC curve analysis, which is consistent with results from a previous study ${ }^{9}$.

In our previous reports, we first introduced the concept of an "HA IgG ANA" that could distinguish between early-stage SLE and SLE that had been active for some time. Our results indicated that the prevalence of HA IgG ANAs was significantly higher in the SLE groups. Notably, the serum concentration of CXCL13 in the HA group was similar to that of the active SLE group and LN group. In addition, we found that the level of serum CXCL13 was positively correlated with the RAI of HA IgG ANAs. Taken together, our results indicated that the high baseline plasma CXCL13 levels reflected the recent onset of inflammation with a better response to early aggressive treatment, which is consistent with a previous study ${ }^{23}$. This could also 
serve as a "window of opportunity" to treat SLE patients with very high CXCL13 levels accordingly.

Our study had several limitations. The experiment contained an insufficient number of people, and we need a larger sample size to confirm our conclusion. Moreover, we did not observe whether different treatments influenced the CXCL13 concentration. Therefore, we intend to study the correlation between treatment with complements C3 and C4 and CXCL13 levels, which requires further study.

In conclusion, our study demonstrated a clinical evaluation of circulation CXCL13 in SLE patients. The concentrations of CXCL13 could be used to identify the disease and the activity of SLE and LN. In addition, CXCL13 might promote the inflammatory process in SLE. Furthermore, HA IgG ANAs might affect the concentration of CXCL13. Therefore, the chemokine CXCL13 might be a risk factor influencing the inflammatory process in SLE.

\section{Authors' contributions}

Conceived and designed the experiments: Y-LZ, YZ,Y-Q L. Performed the experiments: X-L W,Q-G C, Q-H H. Analyzed the data: Y-LZ, YX. Contributed reagents/materials/analysis tools: J-J W,L-C J. Wrote the paper: YX, Y-LZ. Revised the manuscript critically for important intellectual content: Y-LZ. All authors read and approved the final manuscript.

\section{Acknowledgments}

This work was supported by the Natural Science Foundation of Fujian Province (2017J01378).

Data Availability StatementSome or all data, models, or code generated or used during the study are available from the corresponding author by request.

\section{Compliance with ethical standards}

Consent The study was approved by the Institutional Ethics Committee of Zhongshan Hospital, Medical College Xiamen University, and conforms to the ethical guidelines of the Declaration of Helsinki. Requirement for individual patient consent forms was waived due to the retrospective, observational nature of the study.

9.Disclosures The authors report no conflicts of interest.

\section{Reference}

1. Hargraves MM, Richmond H, Morton R. Presentation of two bone marrow elements; the tart cell and the L.E. cell. Proceedings of the staff meetings Mayo Clinic. Jan 21 1948;23(2):25-28.

2. Rosken GHJ, van Beek AA, Bakker-Jonges LE, Schreurs MWJ. Antinuclear antibodies in systemic autoimmune disease. Ned Tijdschr Geneeskd.Apr 23 2020;164.

3. Oliveira RC, Oliveira IS, Santiago MB, Sousa Atta ML, Atta AM. High Avidity dsDNA Autoantibodies in Brazilian Women with Systemic Lupus Erythematosus: Correlation with Active Disease and Renal Dysfunction.J Immunol Res. 2015;2015:814748.

4. Kim HA, Jeon JY, Choi GS, et al. The antichromatin antibodies can be useful as a diagnostic tool and disease activity marker of systemic lupus erythematosus in Koreans. Clin Immunol. Aug 2008;128(2):277283.

5. Fritzler MJ, Salazar M. Diversity and origin of rheumatologic autoantibodies. Clinical Microbiology Reviews. Jul 1991;4(3):256-269.

6. Bao YQ, Wang JP, Dai ZW, et al. Increased circulating CXCL13 levels in systemic lupus erythematosus and rheumatoid arthritis: a meta-analysis. Clin Rheumatol. Jan 2020;39(1):281-290.

7. Niederkorn A, Fruhauf J, Schwantzer G, et al. CXCL13 is an activity marker for systemic, but not cutaneous lupus erythematosus: a longitudinal cohort study. Arch Dermatol Res. Aug 2018;310(6):485-493. 
8. Lee HT, Shiao YM, Wu TH, et al. Serum BLC/CXCL13 Concentrations and Renal Expression of CXCL13/CXCR5 in Patients with Systemic Lupus Erythematosus and Lupus Nephritis. Journal of Rheumatology. Jan 2010;37(1):45-52.

9. Schiffer L, Kumpers P, Davalos-Misslitz AM, et al. B-cell-attracting chemokine CXCL13 as a marker of disease activity and renal involvement in systemic lupus erythematosus (SLE). Nephrol Dial Transplant.Dec $2009 ; 24(12): 3708-3712$.

10. Barone F, Bombardieri M, Rosado MM, et al. CXCL13, CCL21, and CXCL12 expression in salivary glands of patients with Sjogren's syndrome and MALT lymphoma: association with reactive and malignant areas of lymphoid organization. J Immunol. Apr 1 2008;180(7):5130-5140.

11. Bombardier C, Gladman DD, Urowitz MB, Caron D, Chang CH. Derivation of the SLEDAI. A disease activity index for lupus patients. The Committee on Prognosis Studies in SLE. Arthritis Rheum. Jun 1992;35(6):630-640.

12. Hochberg MC. Updating the American College of Rheumatology revised criteria for the classification of systemic lupus erythematosus.Arthritis Rheum. Sep 1997;40(9):1725.

13. Gordon C, Jayne D, Pusey C, et al. European consensus statement on the terminology used in the management of lupus glomerulonephritis.Lupus. Mar 2009;18(3):257-263.

14. Aletaha D, Neogi T, Silman AJ, et al. 2010 Rheumatoid arthritis classification criteria: an American College of Rheumatology/European League Against Rheumatism collaborative initiative. Arthritis Rheum. Sep 2010;62(9):2569-2581.

15. Zeng Y, Lin Y, Wang X, et al. Assessment of a high-avidity IgG ANAs for the diagnosis and activity prediction of systemic lupus erythematosus. Clin Rheumatol. Mar 18 2020;39:2619-2629.

16. Zeng Y-L, Lin Y-Q, Zhang N-N, et al. CXCL13 chemokine as a promising biomarker to diagnose neurosyphilis in HIV-negative patients.Springerplus. Jun 16 2016;5.

17. Lee HT, Shiao YM, Wu TH, et al. Serum BLC/CXCL13 concentrations and renal expression of CXCL13/CXCR5 in patients with systemic lupus erythematosus and lupus nephritis. J Rheumatol. Jan 2010;37(1):45-52.

18. Rioja I, Hughes FJ, Sharp CH, et al. Potential novel biomarkers of disease activity in rheumatoid arthritis patients: CXCL13, CCL23, transforming growth factor alpha, tumor necrosis factor receptor superfamily member 9, and macrophage colony-stimulating factor.Arthritis Rheum. Aug 2008;58(8):2257-2267.

19. Ezzat M, El-Gammasy T, Shaheen K, Shokr E. Elevated production of serum B-cell-attracting chemokine-1 (BCA-1/CXCL13) is correlated with childhood-onset lupus disease activity, severity, and renal involvement.Lupus. Jul 2011;20(8):845-854.

20. Schiffer L, Kielstein JT, Haubitz M, et al. Elevation of serum CXCL13 in SLE as well as in sepsis. Lupus. Apr 2011;20(5):507-511.

21. Kulkarni O, Anders HJ. Chemokines in lupus nephritis. Front Biosci. May 1 2008;13:3312-3320.

22. Moreth K, Brodbeck R, Babelova A, et al. The proteoglycan biglycan regulates expression of the B cell chemoattractant CXCL13 and aggravates murine lupus nephritis. J Clin Invest. Dec 2010;120(12):4251-4272.

23. Greisen SR, Schelde KK, Rasmussen TK, et al. CXCL13 predicts disease activity in early rheumatoid arthritis and could be an indicator of the therapeutic 'window of opportunity'. Arthritis Res Ther. Sep 24 2014;16(5):434.

\section{Hosted file}


table1.pdf available at https://authorea.com/users/371497/articles/489833-the-cxcl13chemokine-serves-as-a-potential-biomarker-to-diagnose-systemic-lupus-erythaematosuswith-disease-activity

\section{Hosted file}

figure1.pdf available at https://authorea.com/users/371497/articles/489833-the-cxcl13chemokine-serves-as-a-potential-biomarker-to-diagnose-systemic-lupus-erythaematosuswith-disease-activity

\section{Hosted file}

figure2.pdf available at https://authorea.com/users/371497/articles/489833-the-cxcl13chemokine-serves-as-a-potential-biomarker-to-diagnose-systemic-lupus-erythaematosuswith-disease-activity

\section{Hosted file}

figure3.pdf available at https://authorea.com/users/371497/articles/489833-the-cxcl13chemokine-serves-as-a-potential-biomarker-to-diagnose-systemic-lupus-erythaematosuswith-disease-activity

\section{Hosted file}

figure4.pdf available at https://authorea.com/users/371497/articles/489833-the-cxcl13chemokine-serves-as-a-potential-biomarker-to-diagnose-systemic-lupus-erythaematosuswith-disease-activity 